\title{
LINGUISTICS AND PSYCHOLOGY
}

\author{
Albert P. Weiss
}

\section{Ohio State University}

For those psychologists for whom psychology is a study of the conditions known as the cultural status of the individual, or the anthropological status of the group, the study of the language mechanisms is taking on a new aspect. Language as a form of behavior through which the individual adjusts himself to a social environment, is not the same thing as language as a medium of expression of so-called subjective desires, hopes, and aspirations. As a form of behavior, language represents biological, physiological, and social conditions; as a medium of expression, it assumes the existence of non-physical forces or types of psychical energy whose existence has not been adequately demonstrated. Therefore, when the psychologist finds himself confronted with the request to make a "psychological" explanation, or a "psychological" interpretation of a careful and detailed linguistic investigation, he is unable to add anything and if anything is added it often only obscures the investigation.

The great gap between the achievements of the modern man and the anthropoid apes or some of the highly socialized bees, wasps, ants, can best be understood as due to the absence of language in the animals. The acuity of man's senses can be matched by those of many animals, his strength and unaided skill is surpassed by others. With respect to vision and audition for instance, the behavior of the lone individual is limited to a space of a few meters in the immediate vicinity of his body or at most to within a few kilometers from the top of a hill or mountain. For the other senses, taste, smell, touch, etc., the range is even less. From a mere consideration of man's position in the scale of biological development, his libraries, museums, telephones, cinema, microscopes, telescopes, and sky-scrapers, seem incomprehensible.

Human achievement, as compared with animal achievement, differentiates itself particularly through its greater variety and through its coöperative character. The essential condition for producing these 
effects is a high degree of sensorimotor interchangeability between individuals. When individuals coöperate, the sense organs and muscles of one act for all; as when the scout announces the approach of the enemy, the eyes of the scout act as substitutes for the eyes of those whom he warns, and his oral report acting on the ears of the warriors releases activities and movements which are as appropriate as if their own eyes had been stimulated by the sight of the enemy. This simple form of sensorimotor interchange is common to both man and animal, and such signaling may be regarded as the beginning of language behavior. In man the process soon becomes very complex. Specific types of external stimuli, in addition to releasing specific manual responses, also release verbal responses, and these become, for other individuals, substitute stimuli for the original stimuli. The number of these substitute systems becomes greater and we have the beginning of what we know as human speech. In addition to such oral substitution, there are developed in the course of time permanent visual substitutes in the form of hieroglyphics, printing, etc., and thus more effective sensorimotor interchangeability between the living and the dead arises. In the evolutionary development of language we approach, as a limit, a condition in which a greater and greater number of the objects and events in the universe (past, present, and future) are represented by substitute language stimuli and their more permanent substitutes exhibiting a wider range of interrelationships than the original objects and events themselves. In this sense language as a form of behavior is the reduction of all the objects and processes of the universe to library dimensions.

To the extent that an individual acquires language, he may be resarded as exhibiting in his behavior, forms of movements (of the gpeech mechanism) which are functionally correlated with those movements which make up the universe. More simply stated, from the psychological point of view, language is that form of behavior known as description. A "description" is a physical event: a series of sound waves which act on the auditory mechanism, a series of light waves reflected from patterns of contrasting surfaces of the type known as written and printed speech, a series of pattern contacts from raised points as in the speech for the blind, the deaf-mute system, and many other forms of representation which need not be enumerated. The language form of behavior makes it possible for a community to act as a single organism in which health is more uniform, life is longer, strength is greater, and the ability to meet unfavorable living condi- 
tions is the best that has been devised. Through language it has been possible to substitute stable inorganic media for the perishable and energy-consuming organic tissues necessary for the maintenance of the proper organization and function of the individual; as when copper telephone wires are substituted for nervous connections. This has greatly enhanced the effectiveness of social organization.

This conception of language leads to a series of problems which have been largely ignored in psychology and in the traditional studies of the language responses.

It may be well to indicate a few language problems common to both linguistics and psychology which need more intensive investigation.

1. Language presents an unlimited variety of responses to external stimuli. The English language of an educated speaker contains many thousand words, each one of which represents a definite stimulus and a definite reaction. When grammatical structure is considered, the variety of possible stimuli and responses is infinite. Is it conceivable that we could by systematic effort modify the English language to give the maximum accuracy to description and the greatest ease in learning?

2. Language behavior virtually unites all the members of a group into a single sensorimotor organization. This brings us to the problem of a universal language. Is an energetic scientific attack on this problem worth while?

3. Language behavior is a device for varying the inherited or the acquired activities of the individual. As a biological function it enormously extends the environment and sensory range of the individual and increases the variability of behavior. Can the parrot-like language habits be replaced by habits in which the individual modifies his language responses in conformity with the progressive alterations in his environment?

4. Language may be either the stimulus for a response or the response for a stimulus. This is at the basis of all the coopperative activities such as industry, law, etc., and the basis of the biosocial and sensorimotor interchangeability between individuals. How can we measure the relative excellencies of various pronunciations? How can those forms which have been found best be introduced? How can our written substitutes be improved?

5. The language response as a substitute stimulus for actual objects and situations makes it possible to reproduce and more critically analyze into its component parts any event that has ever occurred. 
Can the classifications or categories that we thus make, be improved? Can the idea of classification be given greater dominance both in the teaching of language and in the comparative studies of different languages?

6. Words as stimuli for abstractions and generalizations facilitate invention and discovery and form the basis of what is called creative imagination. How do the different languages and the various forms of language structure compare with each other in developing this ability?

7. The language reactions as written or printed records or as the traditions of such relatively permanent institutions as the law and medicine make available the best adjustments that have been invented or discovered. Hơw much of what we call progress in civilization is due to the specific type of language behavior which a nation has developed?

Such considerations suggest themselves readily, and it seems remarkable that different languages and language forms have not been more generally studied by comparative methods with respect to their effectiveness in producing better types of social organization. Many of the so-called psychological interpretations of language are problems which belong to these fields, but one often feels in reading these interpretations that they actually obscure the results which have been secured.

Many of the problems indicated in the preceding classifications can be reduced to experimental dimensions; but experiments of this type are of an order altogether different from the usual studies of language. In psychology they would be classed under learning and habit formation and would become genetic experiments with children, or by the use of specially devised categories of stimuli and responses could become laboratory experiments with adults. Thus language, regarded as a substitute stimulus for the actual objects and events which make up the universe, places the problem of "what is a relation" in a new setting. Those forms of behavior classified as "relative" can only have a word or symbol as a stimulus and the conception "one-half," for instance, is probably impossible without a linguistic symbol as a stimulus. When the more complicated mathematical relationships such as logarithmic, parabolic, infinite series, etc., are considered, language becomes essential. The fact that the symbols for the mathematical relations tend to become uniform as intercommunication between groups of approximately the same cultural status becomes wider, raises 
the general question, Is there a "best" form for grammatical structure, word and sentence structure, and can the question be answered experimentally?

As the sciences of neurology and physíology increase their content and attempt the analysis of man's adjustments to his environment and especially his social environment, there is a growing tendency to enlarge the scope of the physiological factors involved in these adjustments. While mind or consciousness is still accepted as a non-physiological force which in some unknown manner modifies human behavior and determines the upper limit of human achievement, the hypotheses which have been developed as to the nature of mind and how it functions are generally admitted to be unsatisfactory. It has generally been recognized that human behavior exhibits the effects of environmental conditions which are no longer present. To be able, more or less perfectly, to reproduce a pageant in the twentieth century which actually occurred in the twelfth, is so far beyond the capacity of any animal that it seems necessary to postulate a form of energy for man which animals do not possess, or possess only in a very limited degree. That the pageant of the twelfth century is still a part of the environment of the twentieth century in the form of a verbal description in some manuscript, drawing, or other substitute form, is well known, but that this principle alone is sufficient to account for man's greater achievements is not yet clearly recognized.

Further, that such forms of activity as reasoning, the formulation of theories, hypotheses, and complete metaphysical systems, may be the result solely of the development of language behavior as determined by the action of the educational environment on the sensorimotor mechanism of an individual, is not generally recognized by philosophers. For them a system of metaphysics is more than a system of verbal habits which can be completely resolved into the interaction between their animate, inanimate, verbal environment and the type of sensorimotor structure which they inherited. No non-physical, non-biological forces need be postulated, and until it has been conclusively demonstrated that the biological structure of man and his complex language and social environment are unable to produce the social institutions which differentiate him from the animals, the assumption of a special mental force or a mind is gratuitous. As we learn more about language, there arises a tendency to shift the burden of proof as to the existence of a special mental force, upon those who hold this hypothesis. 
To show how language behavior produces (1) a substitute universe practically unlimited by time and space, (2) a type of social organization which can be characterized as a compound multicellular organization, (3) a form of organization in which, as a limiting condition, the sense organs and muscles of any individual may be placed at the disposal of any other individual, (4) a form of behavior that is a function (in the mathematical sense) of every event in the universe that has occurred, is now occurring, or will occur in the future, demands a type of investigator trained in both linguistics and psychology. Such a combination is rare, and it seems to the writer that as the interrelationships of both these sciences are more clearly recognized the inhibiting effect due to the necessity of assuming a non-physical mental energy will be largely removed and the gap between the physical and the social sciences will become narrower. 